\title{
Rol de la colangio pancreatografía retrógrada endoscópica en pacientes con pancreatitis aguda biliar leve y vesícula in situ*
}

\author{
Drs. MARIO ANSELMI M. ${ }^{1}$, ANA MARÍA GEMMATO P. ${ }^{1}$ \\ 1 Departamento de Cirugía Hospital Guillermo Grant B. \\ Facultad de Medicina, Universidad de Concepción. \\ Concepción, Chile.
}

\begin{abstract}
Endoscopic retrograde colangiopancreatography (ERCP) among patients with biliary pancreatitis and gallbladder in situ

Background: An early decompression of the biliary tract can be useful in biliary pancreatitis. This can be accomplished with an ERCP. Aim: To analyze prospectively the outcomes of ERCP and eventual endoscopic management among patients with mild acute biliary pancreatitis with gallbladder in situ. Material and methods: We studied 90 consecutive patients aged between 15 and 92 years ( 71 females) with mild acute pancreatitis and gallbladder in situ, that were subjected to an ERCP and eventual sphincterotomy. Forty nine patients (54\%) had jaundice and $12(13 \%)$ had cholangitis. Results: In 67 patients (74\%), ERCP detected an abnormal ampulla. The bile duct was successfully cannulated in 89 cases $(99 \%)$. Of these, $42(47 \%)$ had a dilated bile duct and $36(40 \%)$ had choledocholithiasis. In two patients (2\%) ERCP was only diagnostic. In the remaining patients, it was therapeutic. Endoscopic treatment was successful in 86 patients $(99 \%)$. No complications arising from the endoscopic procedure were recorded. Follow-up was completed in 84 patients $(94 \%)$. Of these, $10(12 \%)$ were not subjected to a cholecystectomy, in eight due to a high surgical risk and in two due to refusal. In the remaining 74 patients, a cholecystectomy was performed during the same hospitalization in $57(77 \%)$ and on a deferred basis in $17(23 \%)$. Conclusions: In patients with mild acute biliary pancreatitis and gallbladder in situ, ERCP is a safe and effective method.
\end{abstract}

Key words: Biliary pancreatitis, ERCP, gallbladder in situ.

\section{Resumen}

Objetivo: Analizar en forma prospectiva los resultados de la Colangio Pancreatografia Retrógrada Endoscópica (CPRE) y eventual manejo endoscópico en pacientes portadores de pancreatitis aguda biliar (PAB) leve con vesícula in situ. Material y Método: Se estudiaron 90 pacientes consecutivos con PAB leve y vesícula in situ. La edad promedio fue de $49,5 \pm 18,6$ años (78,9\% mujeres y $21,1 \%$ hombres). Del total, 49 pacientes $(54,4 \%)$ presentaron ictericia y $12(13,3 \%)$ colangitis. Resultados: La CPRE pesquisó patología de la ampolla en $67 / 90$ pacientes $(74,4 \%)$. La canulación selectiva de la vía biliar resultó exitosa en

\footnotetext{
* Recibido el 15 de Marzo de 2010 y aceptado para publicación el 18 de Mayo de 2010.

Correspondencia: Dr. Mario Anselmi M.

Casilla 60 C, Concepción, Chile.

manselmi@udec.cl
} 
$89 / 90$ casos $(98,9 \%)$. En estos, se demostró una vía biliar dilatada en 42 (47,2\%) y coledocolitiasis en 36 (40,4\%). La CPRE fue diagnóstica en 2 enfermos $(2,2 \%)$ y hubo indicación de procedimiento terapéutico en los 87 restantes $(97,7 \%)$, este resultó exitoso en $86(98,9 \%)$. No se presentaron complicaciones derivadas de la cirugía endoscópica. Se completó el seguimiento en $84 / 89$ pacientes $(94,4 \%)$. De estos, $10(11,9 \%)$ no fueron colecistectomizados ( 8 debido al riesgo quirúrgico y 2 por rechazo a la intervención). De los 74 restantes, 57 (77\%) fueron sometidos a una colecistectomía dentro de la misma hospitalización y $17(23 \%$ en forma diferida. El 91,9\% de ellas (68/74) mediante laparoscopia. Conclusión: En pacientes con PAB leve y vesícula in situ, la CPRE es un método seguro y efectivo. Además, permite efectuar una colecistectomía por vía laparoscópica, durante la misma hospitalización en la gran mayoría de los pacientes y constituye una alternativa a la colecistectomía en pacientes de alto riesgo quirúrgico.

Palabras clave: Pancreatitis aguda biliar, CPRE, vesícula in situ.

\section{Introducción}

La pancreatitis aguda (PA) afecta entre el 6 y el $8 \%$ de los pacientes con colelitiasis sintomática, aunque puede llegar al $20 \%$ en los enfermos con microlitiasis y hasta un $30 \%$ de aquellos con colesterolosis ${ }^{1}$.

El tránsito de uno o más cálculos desde el colédoco, a través de la papila, hasta el duodeno, parece ser el evento inicial en el desarrollo de una $\mathrm{PA}^{2}$. Algunos estudios han descrito que hasta el $70 \%$ de aquellas pancreatitis que han sido clasificadas inicialmente como idiopáticas, son realmente de origen biliar ${ }^{3,4}$.

Aunque no se sabe con certeza si los cálculos inician la pancreatitis o simplemente contribuyen a su progreso y curso, la alta incidencia de ellos en el colédoco, en las formas más graves, constituyen una base racional para la descompresión biliar precoz en este tipo de pacientes ${ }^{5,6}$.

Algunos estudios clínicos y experimentales han descrito que la duración de la obstrucción biliopancreática se correlaciona con la severidad de la injuria del órgano ${ }^{7}$. Por este motivo, la intervención endoscópica temprana en la PAB se basa en la hipótesis de que la liberación de la obstrucción puede detener el progreso del ataque severo o prevenir el deterioro de un ataque leve $\mathrm{e}^{7-9}$.

El rol de la Colangio Pancreatografía Retrógrada Endoscópica (CPRE) en el manejo de la pancreatitis aguda sigue siendo un tema polémico. Existen controversias sobre si esta debe efectuarse de urgencia en todos los pacientes ${ }^{10,11}$, selectivamente en un subgrupo de enfermos ${ }^{12-15}$, o simplemente no llevarla a cabo ${ }^{7,15}$.

Uno de los principales factores de riesgo de una PAB lo constituye el hecho de tener la vesícula in $s_{i t u^{16}}$. Sin embargo, ya que la gran mayoría de los pacientes con PA tienen un curso benigno, que no es alterado por la CPRE y la esfinterotomía endoscópica (EE), algunos sugieren un uso más selectivo ${ }^{12}$. No obstante, se ha se ha descrito una recidiva de entre 50 y $90 \%$ en aquellos pacientes que no han sido sometidos a una colecistectomía o a una esfinterotomía endoscópica ${ }^{17}$. Esto ha creado un interés creciente en la limpieza endoscópica de la vía biliar antes de la cirugía ${ }^{18}$ para dar la posibilidad de que el paciente sea sometido a una colecistectomía laparoscópica, y por otro lado, para evitar una recidiva de la $\mathrm{PAB}^{18,19-22}$. El objetivo de este trabajo es analizar los resultados de la CPRE y eventual manejo endoscópico, en pacientes portadores de una PAB leve con vesícula in situ.

\section{Material y Método}

Se estudiaron en forma prospectiva 90 pacientes consecutivos que ingresaron con el diagnóstico de PAB leve y vesícula in situ. Para predecir la severidad de la pancreatitis se utilizaron los signos pronósticos de Ranson ${ }^{19}$. Se consideró que la pancreatitis era leve si el paciente presentaba 3 o menos signos pronósticos.

La edad promedio fue de 49,5 $\pm 18,6$ años con un rango que fluctuó entre los 15 y los 92 años. Del total de casos, $71(78,9 \%)$ eran del género femenino y $19(21,1 \%)$ del masculino.

De los 90 sujetos estudiados $61(67,8 \%)$ presentaban evidencias clínicas de obstrucción biliar, 49 $(54,4 \%)$ ictericia y $12(13,3 \%)$ colangitis aguda. El ultrasonido abdominal demostró la presencia de colelitiasis en 76 pacientes $(84,4 \%)$, en $33(36,7 \%)$ reportó una vía biliar dilatada y en 16 enfermos $(17,8 \%)$ se detectó una coledocolitiasis concomitante. El intervalo promedio transcurrido entre el ingreso hospitalario y el procedimiento endoscópico fue de 3,3 \pm 2,5 días (Rango: 0,2-10 días).

Una vez confirmado el diagnóstico y efectuado un estudio de ultrasonido abdominal, los pacientes debieron firmar su consentimiento informado para ser sometidos, de acuerdo al protocolo de trabajo de nuestra Unidad Docente Asistencial de Cirugía Endoscópica (UDACE), a una CPRE y eventual es- 
finterotomía o descompresión endoscópica de la vía biliar (mediante la inserción de una endoprótesis), antes de los 10 días de su ingreso. Lo anterior, con el objeto de prevenir una recidiva del cuadro agudo.

La gran mayoría de los procedimientos fueron realizados con anestesia tópica faríngea (lidocaína al $4 \%$ en spray) y bajo sedación consciente intravenosa con midazolam, en dosis acordes a la edad y condición del paciente. Se utilizó como antiespasmódicos el Butil-bromuro de escopolamina, en dosis de 20 a $40 \mathrm{mg}$ intravenoso. Todos los pacientes durante la CPRE fueron controlados mediante un monitor de signos vitales multiparámetros. Todos los procedimientos terapéuticos fueron efectuados por endoscopistas experimentados.

Los equipos utilizados fueron: Duodenoscopios marca Olympus TJF 145 y JF-V (Tokio Japón). Esfinterótomos marca Olympus (KD-211Q-1520 y/o KD-11Q-1) o Wilson Cook (Canulatome II doble lumen).

Una vez que se obtuvieron los hallazgos de la duodenoscopia y las características de la papila, se procedió a canular selectivamente la vía biliar e inyectar medio de contraste hidrosoluble, para determinar patología biliar, presencia o no de cálculos y sus características de número, forma y tamaño, así como la relación del conducto biliar principal con los mismos. La extracción de los cálculos se realizó con canastillos de Dormia marca Olympus (FG-22Q-1) y la revisión final de la vía biliar con catéteres de balón de triple lumen, marca Olympus (B-231-PAB).

Cuando el procedimiento se consideró técnicamente difícil, o no existían las condiciones necesarias para la extracción de los cálculos, se aseguró la permeabilidad de la vía biliar, mediante la instalación de una prótesis plástica de 7 French, de $8 \mathrm{~cm}$ de longitud, reprogramándose el paciente para un procedimiento definitivo en una nueva oportunidad.

A los enfermos se les hizo seguimiento hasta la solución definitiva de su patología biliar.

\section{Resultados}

Endoscópicamente, el duodeno fue normal en 81 pacientes $(90 \%)$. En $6(6,7 \%)$ se observó la presencia de un divertículo yuxtapapilar. En 2 casos $(2,2 \%)$ la mucosa estaba edematosa y $1(1,1 \%)$ era portador de una úlcera activa.

En 23 casos $(25,6 \%)$ la duodenoscopia demostró una papila de aspecto normal. En los 67 restantes (74,4\%) se observó evidencias de patología ampular. (Tabla 1)

La canulación selectiva de la vía biliar resultó exitosa en 89 de 90 pacientes $(98,9 \%)$ y fracasó en
$1(1,1 \%)$, debido a la posición intradiverticular de la papila. El acceso a la vía biliar fue exitoso mediante la técnica convencional en 67 de 90 casos $(74,4 \%)$, en los 23 restantes $(25,6 \%)$ debió utilizarse un precorte para acceder al colédoco.

En 10 de los 89 casos exitosamente canulados $(11,2 \%)$, la vía biliar resultó normal y en los 79 restantes $(88,8 \%)$ la CPRE pesquisó algún tipo de patología. (Tabla 2). El diámetro promedio de la vía biliar, medido de la placa radiológica de la CPRE,

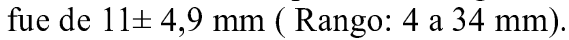

En 35 de los 36 pacientes que presentaron colédocolitiasis $(97,2 \%)$, esta fue múltiple. En 17 de ellos $(47,2 \%)$ se pesquisaron 5 o más cálculos en la vía biliar y en 24 de los 36 casos con cálculos $(66,7 \%)$, estos medían menos de $5 \mathrm{~mm}$ de diámetro.

La CPRE fue diagnóstica en 2 de los 89 enfermos exitosamente canulados $(2,2 \%)$ y hubo indicación de procedimiento terapéutico en los 87 restantes $(97,8 \%)$. De estos últimos, $86(98,9 \%)$ resultaron exitosos y $1(1,1 \%)$ fallido. Tal como en el caso del fracaso en la canulación, debido a que la ubicación intradiverticular de la ampolla, impedía una visión adecuada.

Las indicaciones de la EE se muestran en la Tabla 3. No hubo complicaciones derivadas de la cirugía endoscópica.

Tabla 1. Pancreatitis aguda biliar. Hallazgos del estado de la papila a la duodenoscopia

\begin{tabular}{lcc}
\hline Patología ampular & $\mathbf{n = 9 0}$ & $\mathbf{\%}$ \\
\hline Ostium dilatado & 27 & 30,0 \\
Migración de cálculo & 15 & 16,7 \\
Edema & 11 & 12,2 \\
Cálculo impactado & 6 & 6,7 \\
Ostium estenótico & 6 & 6,7 \\
Fístula suprapapilar & 2 & 2,2 \\
Normal & 23 & 25,6 \\
\hline
\end{tabular}

Tabla 2. Pancreatitis aguda biliar. Hallazgos de la vía biliar a la colangiografía

\begin{tabular}{lcc}
\hline Patología de la vía biliar & $\mathbf{n = 8 9}$ & $\mathbf{\%}$ \\
\hline Dilatada & 42 & 47,2 \\
Colédocolitiasis & 36 & 40,4 \\
Estenosis benigna & 1 & 1,1 \\
Normal & 10 & 11,2 \\
\hline
\end{tabular}


ROL DE LA COLANGIO PANCREATOGRAFÍA RETRÓGRADA ENDOSCÓPICA EN PACIENTES CON PANCREATITIS ...

Tabla 3. Pancreatitis aguda biliar. Indicación de procedimiento terapéutico

\begin{tabular}{lcc}
\hline Indicación & $\mathbf{n = 8 7}$ & $\mathbf{\%}$ \\
\hline Profilaxis de la recidiva & 44 & 50,6 \\
Colédocolitiasis & 36 & 41,4 \\
Divertículo yuxtapapilar & 6 & 6,9 \\
Estenosis benigna & 1 & 1,1 \\
\hline
\end{tabular}

Se completó el seguimiento en 84 de los 89 casos exitosamente canulados $(94,4 \%)$. De estos, 10 $(11,9 \%)$ no fueron colecistectomizados, (8 debido al alto riesgo quirúrgico y 2 por rechazo a la intervención). A los 74 restantes (88,1\%) se les sometió a una colecistectomía. En 57 de estos últimos (77\%) ésta fue efectuada dentro de la misma hospitalización y en $17(23 \%)$ en forma diferida. Fue posible realizar una cirugía laparoscópica en 68 de estos enfermos $(91,9 \%)$. En los casos restantes, la resección de la vesícula biliar fue llevada a cabo mediante cirugía abierta en $5(6,8 \%)$ y se exploró la vía biliar sólo en un paciente $(1,3 \%)$, sin encontrar evidencias de coledocolitiasis.

\section{Discusión}

El rol que la CPRE juega en el manejo endoscópico de la PAB leve sigue generando controversias. Hasta mediados de los ochenta, la CPRE y EE estaban contraindicadas en la PA debido a la posibilidad de agravarla o de inducir un nuevo ataque ${ }^{20}$.

Sin embargo, Neoptolemos en $1988^{11}$ y posteriormente otros autores ${ }^{9,21,22}$, han mostrado que en pacientes con PAB la CPRE con eventual EE es un método seguro. En nuestra serie prospectiva de 90 pacientes consecutivos con PA leve no se presentaron complicaciones derivadas de la cirugía endoscópica.

Sin embargo, como la CPRE y la EE son procedimientos invasivos que pueden dar origen a complicaciones severas ${ }^{23}$, algunos sugieren que debería reservarse a los pacientes con cálculos biliares persistentes.

La ecotomografia abdominal aún se considera el método de elección para el diagnóstico de litiasis de los conductos biliares, no obstante, es altamente operador dependiente ${ }^{24}$, su sensibilidad disminuye considerablemente si los cálculos son pequeños ${ }^{25} \mathrm{y}$ ha mostrado escaso rendimiento en nuestro medio. Emplear métodos más sofisticados y precisos como son la Colangioresonancia Magnética, la Endosonografia, y/o el Ultrasonido Intraductal (IDUS) ${ }^{24,26}$, resultan caros y poco disponibles en la práctica clínica de nuestro país, motivo por el cual hasta la fecha, en nuestra Unidad, la CPRE sigue ocupando lejos, el primer lugar en la pesquisa de litiasis de los conductos biliares.

Se consideró que hubo indicación de cirugía endoscópica en 87 de lo 89 pacientes exitosamente canulados $(97,8 \%)$ y en esta serie, la tasa de morbilidad fue nula, lo que muestra el carácter terapéutico y la seguridad de este procedimiento. No obstante, es procedente aclarar, que todos los procedimientos fueron efectuados por endoscopistas experimentados, en un centro de referencia donde se efectúan un promedio de 1.000 procedimientos anuales, con instrumental de adecuada calidad, personal altamente calificado y con tasas de morbilidad posterior a CPRE tan bajas como el $0,48 \%{ }^{22}$.

Algunos autores sugieren que los pacientes con PA severa, con obstrucción biliar persistente y colangitis asociada podrían considerarse como el único subgrupo que puede beneficiarse de una intervención biliar urgente ${ }^{27}$. Sin embargo, para otros, la CPRE está indicada en el manejo de la PA independiente de la predicción de gravedad de esta $^{28}$, consideran que la EE debería ser el método de elección en todos los casos de PAB ${ }^{10}$.

El tratamiento integral de la litiasis biliar es la mejor alternativa para evitar episodios de PA. En pacientes con una vesícula in situ litiásica que no son sometidos a una colecistectomía o EE, un segundo ataque ocurre entre un $50 \%$ a un $90 \%$ de los casos en un intervalo de pocos meses ${ }^{16}$.

Algunos autores han analizado los factores relacionados con la litiasis y la anatomía pancreatobiliar que predisponen a una $\mathrm{PA}^{15}$, lo que resultaría adecuado considerar. Por otra parte, es de suponer que tras la EE las condiciones anatómicas que favorecen la obstrucción del flujo exocrino pancreático deberían desaparecer ${ }^{29}$.

Los datos que confirman esta hipótesis se extrajeron del análisis secundario de trabajos en los que, con el fin de evitar la cirugía en pacientes con alto riesgo quirúrgico, se realizó una EE y limpieza de la vía biliar pero dejando la vesícula litiásica in situ. Se han publicado 6 trabajos prospectivos no controlados que estudian este aspecto ${ }^{30-35}$. Los resultados son bastante satisfactorios ya que las tasas de recidiva a largo plazo fluctuaron entre un $0 \%$ y un $5,2 \%$.

En este estudio, se encontró una tasa de colédocolitiasis de 40,4\%. En estos pacientes no hay dudas respecto a la indicación de una EE. En el $60 \%$ restante y de acuerdo al protocolo de trabajo, la sección de la papila se llevó a cabo para prevenir una nueva obstrucción biliar y la posibilidad de recidiva de la $\mathrm{PA}$.

Aunque la colecistectomía se efectúe precozmen- 
te después del cuadro agudo, la manipulación operatoria puede dar origen al paso de cálculos desde la vesícula a la vía biliar ${ }^{15}$.

Se considera que la EE juega un rol importante en la prevención de la recidiva de la $\mathrm{PAB}$ en pacientes con vesícula in situ ${ }^{36}$. La demora que con frecuencia se produce entre el cuadro agudo y la colecistectomía, se asocia a una alta tasa de recidiva de la pancreatitis, salvo que el paciente esté protegido mediante una esfinterotomía previa ${ }^{35,37}$. Incluso en algunos casos seleccionados de pacientes con vesícula in situ, la CPRE + EE puede constituir el único tratamiento a largo plazo para $\mathrm{PAB}^{38-40}$. Finalmente, el barro biliar y la microlitiasis, que habitualmente pasan desapercibidos a los estudios habituales, se cuentan como posibles causas de pancreatitis "idiopáticas" recurrentes ${ }^{3,4}$, que no deberían suceder de existir una amplia comunicación entre la vía biliar y el duodeno ${ }^{29}$.

Por último, en ausencia de la posibilidad de manejo laparoscópico de los cálculos coledocianos, la limpieza preoperatoria del hepatocolédoco permite efectuar una colecistectomía por esta vía durante la misma hospitalización. En estos casos el paciente se encuentra protegido contra la migración intraoperatoria de nuevos cálculos al colédoco.

Como lo demuestran los resultados de este trabajo, fue posible efectuar la colecistectomía durante la misma hospitalización en 3 de cada 4 pacientes y en casi el $92 \%$ por vía laparoscópica. Al término del seguimiento, incluso en los pacientes no colecistectomizados, no se registraron complicaciones ni recidiva del cuadro pancreático.

En pacientes con pancreatitis biliar leve y vesícula in situ, la CPRE es un método seguro y efectivo que no adiciona morbilidad. Permite efectuar una colecistectomía por vía laparoscópica durante la misma hospitalización en la gran mayoría de los pacientes y provee además una alternativa a la resección de la vesícula como prevención de la PAB en pacientes no aptos para el tratamiento quirúrgico.

\section{Referencias}

1. Neoptolemos JP, Stonelake P, Radley S. Endoscopic sphincterotomy for acute pancreatitis. Hepatogastroenterology 1993;40:550-5.

2. Acosta J, Ledesma C. Gallstone migration as a cause of acute pancreatitis. N Engl J Med. 1974;290:448-87.

3. Lee SP, Nicholls JF, Park HZ. Biliary sludge as a cause of acute pancreatitis. N Engl J Med. 1992;326:589-93.

4. Ros E, Navarro S, Bru C, García-Puges A, Valderrama R. Occult microlithiasis in "idiopathic" acute pancreatitis: prevention of relapses by cholecystectomy or ursodexycholic acid therapy. Gastroenterology 1991;101:1701-9.
5. Neoptolemos JP. The theory of "persisting" common bile duct stones in severe gallstone pancreatitis. Ann R Coll Surg Engl. 1989;71:326-31.

6. Oría A, Cimmino D, Ocampo C, Silva W, Kohan G, Zandalazini H, et al. Early endoscopic intervention versus early conservative management in patients with acute gallstone pancreatitis and biliopancreatic obstruction: a randomized clinical trial. Ann Surg. 2007; 245:10-7.

7. Rünzi V, Salija AK, Lerch MM, Dawra R, Nishino H, Steer ML. Early ductal descompression prevents the progression of biliary pancreatitis: an experimental study in the opossum. Gastroenterology 1993;105:157-64.

8.- Acosta JM, Rubio Galli OM, Rossi R, Chinellato AV, Pellegrini CA. Effect of duration of ampullary gallstone obstruction on severity of lesion of acute pancreatitis. J Am Coll Surg. 1997;183:499-505.

9. Fan ST, Lai EC, Mok FP, Lo CM, Zheng SS, Wong J. Early treatment of acute biliary pancreatitis by endoscopic papillotomy. N Engl J Med. 1993;4:228-32.

10. Nowak A, Nowakowska-Dculawa E, Marek TA, Rybicka J. Final results of the prospective randomized controlled study on endoscopic sphicterotomy versus conventional management in acute biliary pancreatitis. Gastroenterology 1995; 108:A380.

11. Neoptolemos JP, Carr-Locke DL, London NJ, Bailey IA, James D, Fossard DP. Controlled trial of urgent endoscopic retrograde cholangiopancreatography versus conservative treatment for acute pancreatitis due to gallstones. Lancet 1988;2:979-83.

12.- De Virgilio C, Verbin C, Chang L, Linder S, Stabile BE, Klein S. Gallstone Pancreatitis. The role of preoperative endoscopic retrograde cholangiopancreatography. Arch Surg. 1994;129:909-13.

13. Young YP, Lo SF, Yip AW. Role of ERCP in the management of predicted mild acute biliary pancreatitis. Asian J Surg. 2003;26:197-201.

14. Fölsch UR, Nitsche R, Lüdtke R, Hilgers RA, Creutzfeldt W, and the German Study Group on Acute Biliary Pancreatitis. Early ERCP and papillotomy compared with conservative treatment for acute biliary pancreatitis. N Engl J Med. 1997;336:237-42.

15. Sugiyama M, Atomi Y. Risk factors for acute biliary pancreatitis. Gastrointest Endosc. 2004;60:210-2.

16. Kelly TR, Swaney PE. Gallstone pancreatitis. The second time around. Surgery 1982;92:571-5.

17. Heider TR, Brown A, Grimm IS, Behrns KE. Endoscopic sphincterotomy permits interval laparoscopic cholecystectomy in patients with moderately severe gallstone pancreatitis. J Gastrointest Surg. 2006;10:1-5.

18. Uhl W, Warshaw A, Imrie C, Bassi C, McKay CJ, Lankisch PG, et al. Guidelines for the Surgical Management of Acute Pancreatitis. Pancreatology 2002;2:565-73.

19. Ranson JHC, Ethiological and prognosis factors in human acute pancreatitis: a review. Am J Gastroenterol. 1982;77:623-6.

20. Sheurer U. Acute pancreatitis: ERCP/Endoscopic papi- 
llotomy yes or no? Swiss Surg. 2000;6:246-8.

21. Loperfido S, Angelini G, Benedetti G, Chilovi F, Costan F, De Berardinis F, et al. Major early complications from diagnostic and therapeutic ERCP: a prospective multicenter study. Gastrointest Endosc. 1998;48:1-10.

22. Devuni D, Aghenta A, Kothari T, Rapson A. Complication rate of gastrointestinal endoscopy procedures at a community hospital. Gastrointestinal Endoscopy. 2008;67:A237.

23. Freeman ML. Adverse outcomes of ERCP. Gastrointest Endosc. 2002;56:273-82.

24. Gandolfi L, Torresan F, Solmi L, Puccetti A. The role of ultrasound in biliary and pancreatic diseases. Eur $\mathrm{J}$ Ultrasound 2003;16:141-59.

25. Sugiyama M, Atomi Y. Acute biliary pancreatitis: the roles of endoscopic ultrasonography and endoscopic retrograde cholangiopancreatography. Surgery 1998; 124:14-21.

26. Moon JH, Cho YD, Cha SW, Cheon YK, Ahn HC, Kim YS, et al. The detección of bile duct stones in suspected biliary pancreatitis: comparison of MRCP, ERCP and intraductal US. Am J Gastroenterol. 2005;100:1051-7.

27. Bornman PC, Beckingham IJ, Krige JE. Gallstone pancreatitis- a critical review of current treatment strategies. S Afr J Surg. 2000;38:97-9.

28. Fan ST, Lai ECS, Mok FPT, Lo Chung-Mau, Zheng S, Wong J. Early treatment of acute biliary pancreatitis by endoscopic papillotomy. N Engl J Med. 1993;328:22832.

29. Carter DC. Gallstone Pancreatitis. Br J Med. 1983;23: 1303-4.

30. Targarona EM, Pros I, Martínez J, Trías M, Ayuso RMP, Ros E, et al. Randomised trial of endoscopic sphincterotomy with gallbladder left in situ versus open surgery for common bileduct calculi in high-risk patients. Lan- cet 1996;347:926-929.

31. Siegel JH, Veerappan A, Cohen SA, Kasmin FE. Endoscopic sphintcterotomy for biliary pancreatitis: an alternative to cholecystectomy in high-risk patients. Gastrointest Endosc. 1994;40:573-5.

32. Wellbourn CRB, Beckly DE, Eyre-Brook IA. Endoscopic sphincterotomy without cholecystectomy for gallstone pancreatitis. Gut 1995;37:119-20.

33. Hervás AJ, Naranjo A, Dios JF, Miño G. Prevención de la recidiva de la pancreatitis aguda biliar mediante esfinterotomía endoscópica. Gastroenterol Hepatol. 1996;19:445-7.

34. Uomo G, Manes G, Lacceti M, Cavallera A, Rabitti PG, et al. Endoscopic sphincterotomy and recurrence of acute pancreatitis in gallstone patients considered unfit for surgery. Pancreas 1997;14:28-31.

35. Gislasonn H, Vetrhus M, Horn A, Hoem D, Söndenaa K, Soreide O, et al. Endoscopic sphincterotomy in acute gallstone pancreatitis: a prospective study of the late outcome. Eur J Surg. 2001;167:204-208.

36. Hervás AJ, Reyes A, Sánchez TZ. Pancreatitis Aguda y CPRE. Rev And Pat Digest. 2002;5:108-118.

37. Kaw M, Al-Antably Y, Kaw P. Management of gallstone pancreatitis: Cholecistectomy or ERCP and endoscopic spchincterotomy. Gastrointest Endosc. 2002;56:6165 .

38. Winslet MC, Imray C, Neoptolemos JP. Biliary acute pancreatitis. Hepatogastroenterology 1991;38:120-123.

39. Neoptolemos JP. Endoscopic sphincterotomy in acute gallstone pancreatitis. Br J Surg. 993;80:547-9.

40. Siddiqui A, Mitroo P, Kowalski T, Loren D. Endoscopicc sphincterotomy with or without cholecystectomy for choledocholithiasis in high-risk surgical patients: a decision analysis. Aliment Pharmacol Ther. 2006;24:1059-66. 